\title{
Quando Tudo dá Errado
}

\section{When Everything goes Wrong}

\author{
Helder Santos, ${ }^{1}$ Hugo Miranda, ${ }^{1}$ Mariana Santos, ${ }^{1}$ Inês Almeida, ${ }^{1}$ Joaquim Peixoto, ${ }^{2}$ Joana Chin, ${ }^{1}$ Lurdes Almeida $^{1}$ \\ Departamento de Cardiologia, Centro Hospitalar Barreiro Montijo E.P.E., ${ }^{1}$ Barreiro - Portugal \\ Departamento de Medicina Interna, Centro Hospitalar Barreiro Montijo E.P.E., ${ }^{2}$ Barreiro - Portugal
}

\section{Introdução}

O procedimento de Bentall foi descrito pela primeira vez há 50 anos e passou por várias melhorias ao longo dos anos. Essa técnica é considerada um procedimento seguro e antigo. Porém, como qualquer cirurgia, pode apresentar várias complicações, como pseudoaneurismo anastomótico, infarto do miocárdio e endocardite. ${ }^{1}$

Em países desenvolvidos, com acesso diferenciado a cuidados de saúde e profilaxia, a endocardite é uma patologia incomum, associada a complicações frequentes e altas taxas de mortalidade. A antibioticoterapia visa erradicar o microrganismo responsável. ${ }^{2}$ No entanto, alguns dos medicamentos utilizados causam diversos efeitos colaterais, como a síndrome DRESS (Reação a Medicamentos com Eosinofilia e Sintomas Sistêmicos).

A síndrome DRESS foi descrita pela primeira vez por Bocquet et. al. em 1996, em pacientes com sintomas constitucionais, linfadenopatia e eosinofilia periférica. É considerada uma reação idiossincrática importante e de hipersensibilidade a medicamentos, com extensas características clínicas. Sua incidência é desconhecida, mas ocorre com maior frequência em adultos. ${ }^{3,4}$ Vários medicamentos estiveram associados à DRESS, mas a vancomicina é um dos mais frequentes. ${ }^{3,5} \mathrm{~A}$ DRESS tem um amplo espectro de condições clínicas, desde sintomas leves até falência de múltiplos órgãos. No entanto, o tempo de exposição ao medicamento, a suscetibilidade individual e o diagnóstico imediato podem influenciar a resposta do paciente. As taxas de mortalidade variam de 3 a $10 \%$ e o diagnóstico imediato e a retirada do medicamento são importantes para obter um resultado favorável. ${ }^{3,4}$

Os autores apresentam um caso único que reflete um conjunto de eventos esporádicos que ocorreram em um paciente.

\section{Palavras-chave}

Síndrome Coronariana Aguda; Doenças da Valva Aorta/ cirurgia; Endocardite; Antibioticoprofilaxia; Síndrome de Hipersensibilidade a Medicamento; Complicações Pós-Operatórias.

\section{Correspondência: Helder Santos •}

Departamento de Cardiologia, Centro Hospitalar Barreiro-Montijo -

Avenida Movimento das Forças Armadas, 2834-003. Barreiro - Portugal

E-mail: helder33689@gmail.com

Artigo recebido em 25/12/2019, revisado em 27/04/2020, aceito em 10/06/2020

DOI: https://doi.org/10.36660/abc.20190907

\section{Relato de Caso}

O paciente é um homem de 60 anos de idade, com histórico clínico de hipertensão arterial, dislipidemia e procedimento de Bentall 8 meses antes da internação, com implante de valva aórtica mecânica St. Jude e Uni-Graft aórtico de 28 mm devido a aneurisma de aorta ascendente (56 mm).

Na sala de emergência, o paciente apresentou dispneia, fadiga, cansaço e sudorese. O exame físico revelou frequência cardíaca de 120 bpm, pressão arterial de 170/94 mmHg, estertores pulmonares e edema periférico. Os exames de sangue revelaram anemia e elevação dos biomarcadores de necrose miocárdica. O eletrocardiograma (ECG) mostrou ritmo sinusal, bloqueio de ramo direito, inversão da onda $\mathrm{T}$ de $0,05 \mathrm{mV}$ em $\mathrm{Dl}$ e aVL e infradesnivelamento do segmento ST de $0,1 \mathrm{mV}$ de $\mathrm{V} 4$ a V6. A ecocardiografia transtorácica revelou válvula mecânica aórtica normofuncionante com leve vazamento protésico e função ventricular esquerda preservada. O paciente apresentou episódios recorrentes de edema pulmonar agudo durante a internação. Em um desses episódios, foram identificadas alterações dinâmicas ao ECG e novo aumento de biomarcadores cardíacos. O paciente evoluiu com choque cardiogênico com nova disfunção sistólica ventricular esquerda e hipocinesia difusa. A angiocoronariografia descartou doença arterial coronariana e, no entanto, revelou compressão extrínseca da artéria coronária esquerda, sugerindo pseudoaneurisma entre o Uni-Graft e a valva aórtica mecânica que comprimia a artéria coronária esquerda. Esse achado foi confirmado em angiotomografia cardíaca (Figura 1).

O paciente foi submetido a ressecção de pseudoaneurisma e substituição da valva aórtica mecânica. Durante a cirurgia, foram identificadas imagens de vegetação sugestivas de endocardite infecciosa. Iniciou-se tratamento empírico com flucloxacilina, vancomicina, ceftriaxona e rifampicina, com hemocultura negativa e resposta inicial favorável.

No $24^{\circ}$ dia de antibioticoterapia, o paciente apresentou febre súbita associada a exantema maculopapular não confluente e não pruriginoso no abdome, membros superiores e inferiores e tórax, além de linfadenopatias. Inicialmente, admitiu-se toxicidade por rifampicina, sendo o medicamento suspenso com recuperação clínica gradual.

No entanto, 12 dias depois, o paciente apresentou quadro clínico semelhante com erupção cutânea (Figuras 2 e 3), febre, linfocitose com dismorfia nuclear, eosinofilia, hepatite aguda, lesão renal aguda e estados alterados de consciência (oscilações entre confusão mental e obnubilação). A deterioração da evolução clínica ocorreu rapidamente, exigindo ventilação invasiva e suporte com vasopressor. A tomografia computadorizada de crânio, tórax e abdome não mostrou achados patológicos. Repetiu-se o ecocardiograma transtorácico e a função da válvula protética estava normal. A punção lombar exibiu resultados normais. As hemoculturas, cultura da valva mecânica, os 


\section{Relato de Caso}

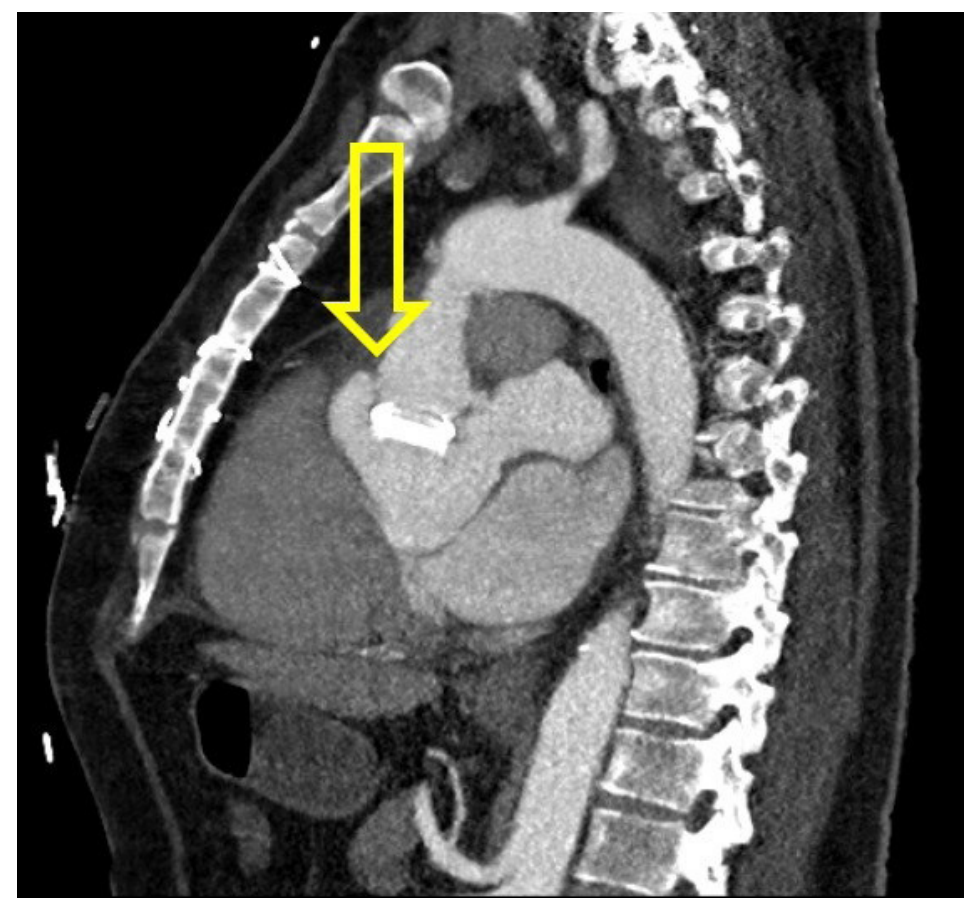

Figura 1 - A angiotomografia cardiaca SACAR revelou compressão extrínseca da artéria coronária esquerda secundária ao pseudoaneurisma entre o Uni-Graft e a valva aórtica mecânica.

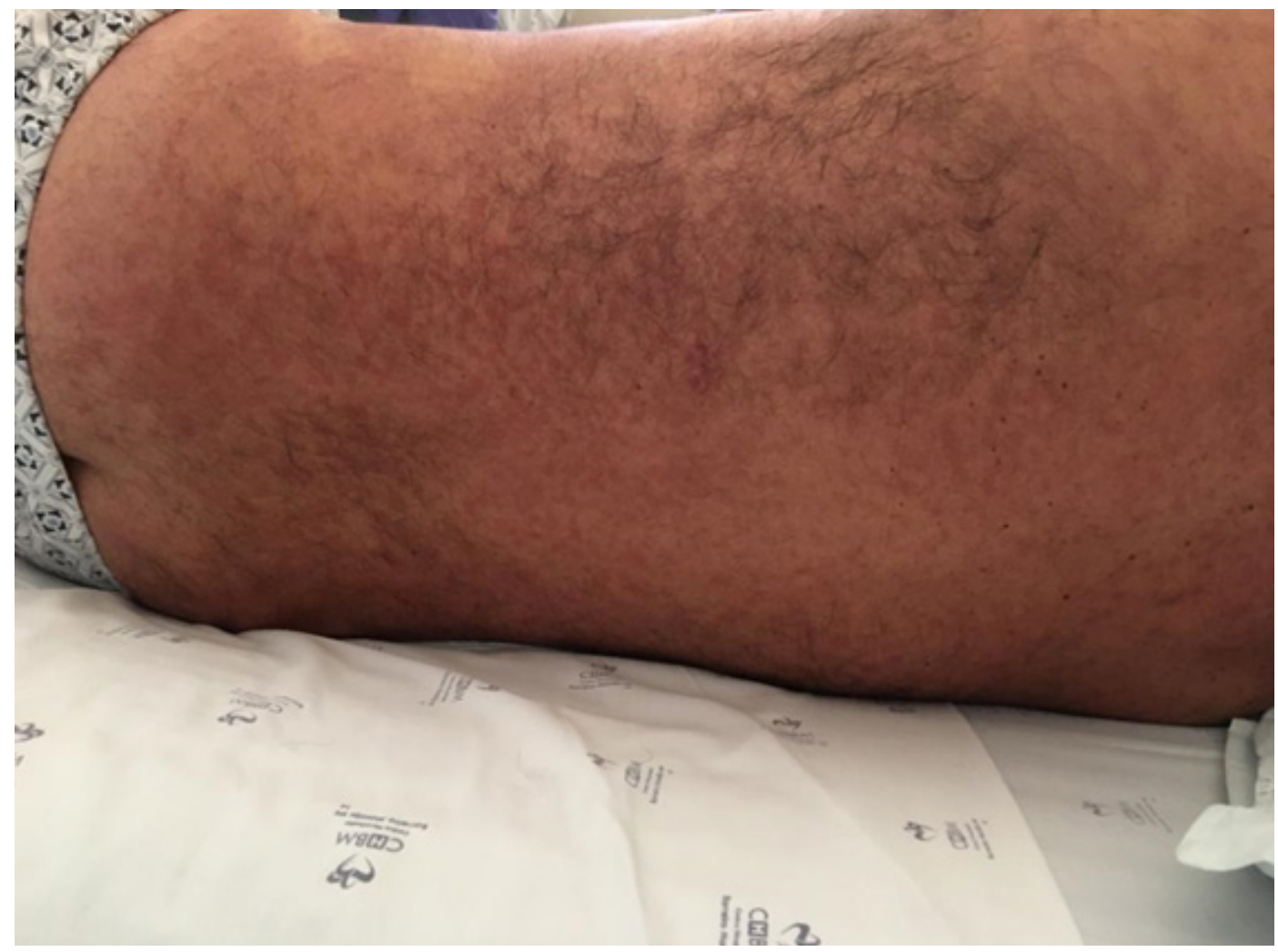

Figura 2 - Erupção cutânea maculopapular não confluente e não pruriginosa no tórax e no dorso. 


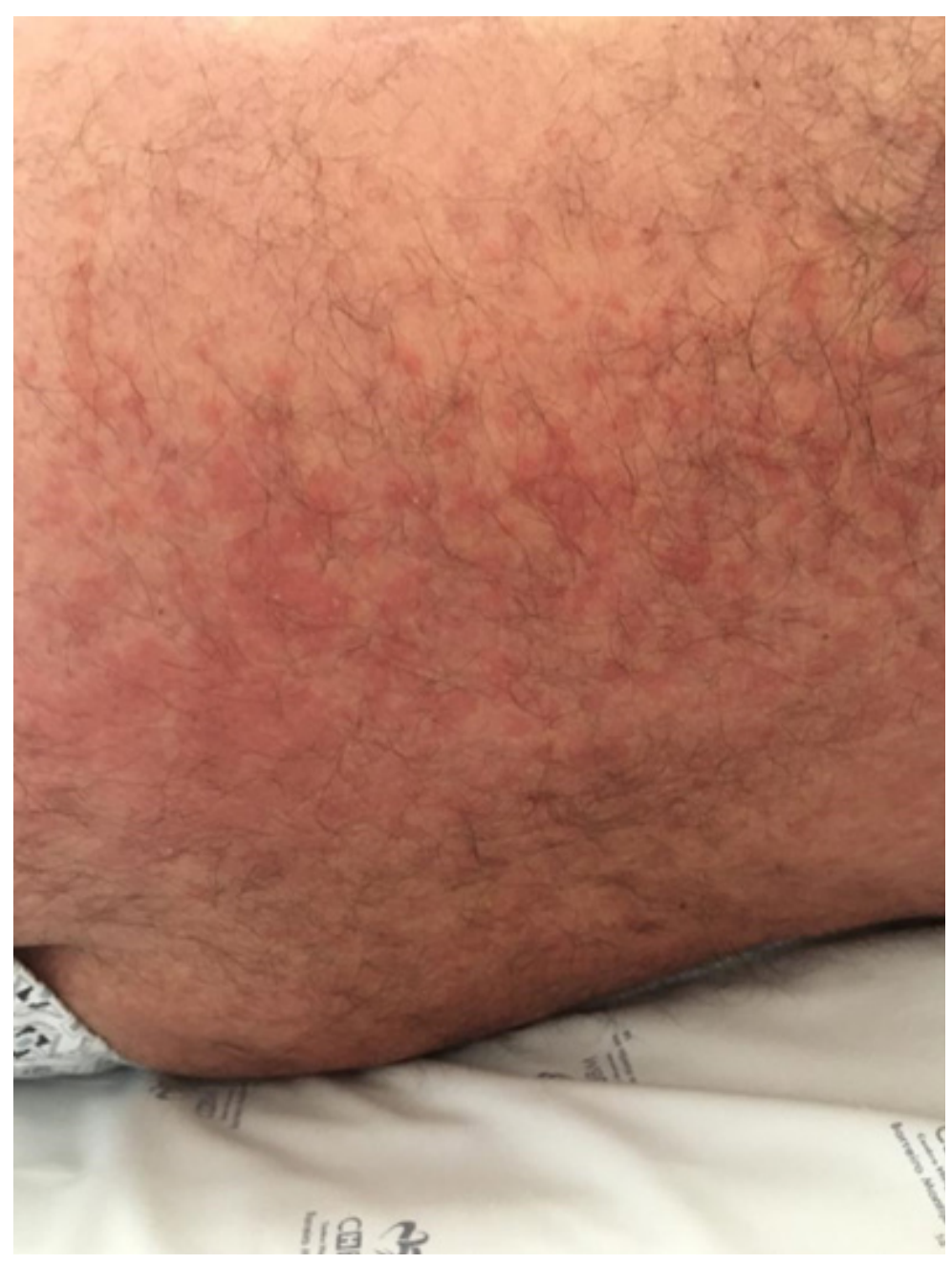

Figura 3 - Erupção cutânea maculopapular não confluente e não pruriginosa no dorso.

testes sorológicos (exceto para herpes zoster) e os testes de autoimunidade foram negativos. A biópsia de pele revelou reação inflamatória. Após descartar outras patologias por meio de uma investigação exaustiva, supôs-se a hipótese de síndrome de DRESS secundária à vancomicina. A retirada da vancomicina juntamente com suporte de terapia intensiva e altas doses de corticoterapia levaram à melhora gradual da função dos órgãos do paciente. Em um ano de seguimento, não houve nenhuma complicação ou déficit.

\section{Discussão}

Problemas técnicos no procedimento de Bentall podem promover deiscências, que podem levar a pseudoaneurisma anastomótico. O local de deiscência e as estruturas circundantes podem levar a eventos cardiovasculares. ${ }^{1,6}$

Países desenvolvidos apresentam incidência relevante de endocardite valvar protética e hemoculturas são o padrão-ouro para o diagnóstico. ${ }^{2}$ Os critérios de Duke modificados fornecem um diagnóstico padronizado e devem ser aplicados com cuidado na endocardite infecciosa. Quanto à endocardite de prótese valvar, os critérios de Duke modificados apresentam menor acurácia diagnóstica. O caso relatado apresentava dois critérios menores: febre e cirurgia cardíaca prévia. De acordo com os critérios de Duke, três critérios menores são necessários para uma possível endocardite. ${ }^{2}$ No entanto, optamos por supor que o diagnóstico e o tratamento empírico foram iniciados, mesmo na presença de culturas negativas do tecido valvar ressecado.

A patogenia da síndrome de DRESS é pouco conhecida. No entanto, é globalmente aceita a interação entre diferentes mecanismos, como predisposições genéticas do paciente, anormalidades metabólicas que levam ao acúmulo de metabólitos de medicamentos e interações medicamentos-vírus que levam à reativação dos herpes-vírus humano (HHV) 6 e 7. As manifestações clínicas aparecem após um longo período de exposição ao medicamento e consistem em erupções cutâneas, alterações hematológicas, linfadenopatias e disfunção multissistêmica. ${ }^{3}$ Se houver suspeita de DRESS, recomenda-se o teste para HHV, pois a infecção pelo HHV está relacionada a maiores complicações e maior tempo de internação. ${ }^{7}$ 
O projeto RegiSCAR (Registro Europeu de Reações Cutâneas Adversas Graves a medicamentos e coleta de amostras biológicas) sugere que pelo menos três dos seguintes critérios são necessários para o diagnóstico: hospitalização, febre, suspeita de reação a medicamentos, erupção cutânea aguda, linfadenopatias em duas áreas diferentes, disfunção orgânica e anormalidades sanguíneas. ${ }^{8}$ De acordo com o SCAR-J (grupo japonês de reações adversas cutâneas graves a medicamentos), ${ }^{9}$ o diagnóstico é estabelecido pela presença dos cinco critérios a seguir: erupção maculopapular após três semanas de tratamento, febre, linfadenopatias, leucocitose, hepatite e reativação do vírus HHV 6. Portanto, nosso paciente exibiu 6 critérios RegiSCAR para o diagnóstico de DRESS. Ainda assim, usando os critérios da SCAR-J, nosso paciente não atende a todos os requisitos para o diagnóstico de DRESS, uma vez que não foi detectada reativação do vírus HHV 6, sendo classificado como apresentação atípica de DRESS.

As recomendações atuais para orientar o tratamento da síndrome DRESS baseiam-se em relatos de casos e opinião de especialistas, e todas preconizam a suspensão imediata do medicamento responsável e, se possível, redução dos outros. Além disso, a corticoterapia geralmente é usada. No entanto, não existem estudos que revelem qualquer eficácia clara e alguns autores defendem que pode exacerbar a reativação viral. Pacientes com DRESS devem ter acompanhamento de longo prazo, pois apresentam maior risco de doenças autoimunes. ${ }^{10}$

A endocardite é uma complicação frequente em pacientes submetidos à cirurgia cardíaca. O uso de vancomicina tem aumentado nos últimos anos e, portanto, está mais frequentemente associado à síndrome DRESS. Como as manifestações clínicas e as anormalidades laboratoriais são inespecíficas, o diagnóstico de DRESS depende da suspeita clínica precoce. O rápido reconhecimento e identificação da síndrome DRESS são essenciais para uma abordagem terapêutica eficaz e baixas taxas de mortalidade.

\section{Agradecimentos}

Os autores agradecem a todos os profissionais do Centro Hospitalar Barreiro-Montijo EPE pela ajuda na publicação deste caso clínico.

\section{Contribuição dos Autores}

Concepção e desenho da pesquisa, Análise e interpretação dos dados e Revisão crítica do manuscrito quanto ao conteúdo intelectual importante: Santos H, Miranda H, Santos M, Almeida I, Chin J, Almeida L; Obtenção de dados: Santos H, Miranda H, Santos M, Chin J; Análise estatística: Santos $\mathrm{H}$, Santos M; Redação do manuscrito: Santos H, Miranda H, Santos M, Almeida I, Chin J.

\section{Potencial Conflito de Interesses}

Declaro não haver conflito de interesses pertinentes.

\section{Fontes de Financiamento}

O presente estudo não teve fontes de financiamento externas.

\section{Vinculação Acadêmica}

Não há vinculação deste estudo a programas de pós-graduação.

\section{Aprovação Ética e Consentimento Informado}

Este artigo não contém estudos com humanos ou animais realizados por nenhum dos autores.

\section{Referências}

1. Sioris T, David TE, Ivanov J, Armstrong S, Feindel CM. Clinical outcomes after separate and composite replacement of the aortic valve and ascending aorta. J Thorac Cardiovasc Surg.2004;128(2):260-5.

2. Habib G, Lancellotti P, Antunes MJ, Bongiorni MG, Casalta J-P, Del Zotti F, et al. 2015 ESC guidelines for the management of infective endocarditis: the task force for the management of infective endocarditis of the European Society of Cardiology (ESC) endorsed by: European Association for CardioThoracic Surgery (EACTS), the European Association of Nuclear Medicine (EANM). Eur Heart J. 2015;36(44):3075-128.

3. Cacoub P, Musette P, Descamps V, Meyer O, Speirs C, Finzi L, et al. The DRESS syndrome: a literature review. Am J Med. 2011;124(7):588-97.

4. Husain Z, Reddy BY, Schwartz RA. DRESS syndrome: Part I. Clinical perspectives. J Am Acad Dermatol. 2013;68(5):693. e1- e14.

5. Kardaun S, Sekula P, Valeyrie-Allanore L, Liss Y, Chu C, Creamer D, et al. Drug reaction with eosinophilia and systemic symptoms (DRESS): an original multisystem adverse drug reaction. Results from the prospective RegiSCAR study. Br J Dermatol. 2013;169(5):1071-80.

6. Haddy SM. Aortic pseudoaneurysm after Bentall procedure. JCardiothorac Vasc Anesth. 1999;13(2):203-6.

7. Tohyama M, Hashimoto K, Yasukawa M, Kimura H, Horikawa T, Nakajima $K$, et al. Association of human herpesvirus 6 reactivation with the flaring and severity of drug-induced hypersensitivity syndrome. Br J Dermatol. 2007;157(5):934-40.

8. Kardaun S, Sidoroff A, Valeyrie-Allanore L, Halevy S, Davidovici B, Mockenhaupt M, et al. Variability in the clinical pattern of cutaneous sideeffects of drugs with systemic symptoms: does a DRESS syndrome really exist? BrJ Dermatol. 2007;156(3):609-11.

9. Shiohara T, lijima M, Ikezawa Z, Hashimoto K. The diagnosis of a DRESS syndrome has been sufficiently established on the basis of typical clinical features and viral reactivations. Br J Dermatol. 2007;156(5):1083-4.

10. Chen Y-C, Chang C-Y, Cho Y-T, Chiu H-C, Chu C-Y. Long-term sequelae of drug reaction with eosinophilia and systemic symptoms: a retrospective cohort study from Taiwan. J Am Acad Dermatol. 2013;68(3):459-65. 